\title{
Analytical and Solutions of Fourth Order Difference Equations
}

\author{
Marwa M. Alzubaidi ${ }^{1,2 *}$ and E. M. Elsayed ${ }^{1,3}$
}

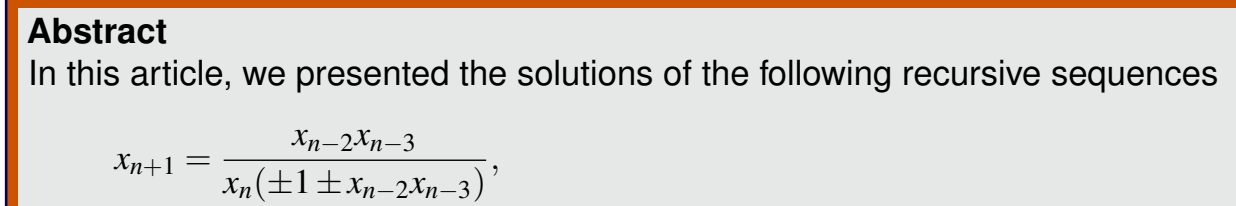

where the initial conditions $x_{-3}, x_{-2}, x_{-1}$ and $x_{0}$ are arbitrary real numbers. Also, we studied some dynamic behavior of these equations.

Keywords: Difference equation, Stability, Global attractor, Linearized stability, Periodicity

2010 AMS: P39A10

${ }^{1}$ King Abdulaziz University, Faculty of Science, Mathematics Department, P. O. Box 80203, Jeddah 21589, Saudi Arabia.

${ }^{2}$ Mathematics Department, The University College of Duba, University of Tabuk, Saudi Arabia

${ }^{3}$ Mansoura University, Mansoura 35516, Egypt. Department of Mathematics, Faculty of Science

*Corresponding author: mmialzubaidi@hotmail.com

Received: 25 July 2018, Accepted: 12 November 2018, Available online: 22 March 2019

\section{Introduction}

Recently, there has been an increasing interest in the study of global behavior of rational difference equations. The reason behind that is because difference equations appear naturally as discrete analogues and as numerical solutions of differential and delay differential equations having applications in biology, ecology, physics, etc. See [1]. Rational difference equations is an important class of difference equations where they have many applications in real life, for example, the difference equation $x_{n+1}=\frac{a+b x_{n}}{c+x_{n}}$, which is known by Riccati Difference Equation has an application in optics and mathematical biology. For more results of the investigation of the rational difference equation see ([2]-[36]) and the references therein.

Karatas [37] examined the global behavior of higher order difference equation

$$
x_{n+1}=\frac{a x_{n-(2 k+1)}}{b+c x_{n-2 k} x_{n-(2 k+1)}} .
$$

In [38] Gumus et al. studied behavior of a third order difference equation

$$
x_{n+1}=\frac{\alpha x_{n}}{\beta+\gamma x_{n-1}^{p} x_{n-2}^{q}} .
$$

Elsayed [39] investigated the global of a higher order rational difference equation 


$$
x_{n+1}=a+\frac{b x_{n-l}+c x_{n-k}}{d x_{n-l}+e x_{n-k}} .
$$

In [40], Kulenovic has got the global stability, periodic nature and gave the solution of non-linear difference equation

$$
x_{n+1}=\frac{\alpha x_{n}+\beta x_{n-1}}{A+B x_{n-1}} .
$$

Elsayed [41] obtained periodic solution of period two and three of the difference equation

$$
x_{n+1}=\alpha+\frac{\beta x_{n}}{x_{n-1}}+\frac{\gamma x_{n-1}}{x_{n}} .
$$

Al-Shabi and Abo-Zeid [42] studied the global stability, periodic and boundedness of the positive solutions of the difference equation

$$
x_{n+1}=\frac{A x_{n-2 r-1}}{B+C x_{n-2 l} x_{n-2 k}} .
$$

Amleh and Drymonis [43] investigated the global character of solution of a certain rational difference equation

$$
x_{n+1}=\frac{\left(\alpha x_{n}+\beta x_{n} x_{n-1}+\gamma x_{n-1}\right) x_{n}}{A x_{n}+B x_{n} x_{n-1}+C x_{n-1}}
$$

Nirmaladevi and Karthikeyan [44] studied periodicity solution and the global stability of nonlinear difference equation

$$
y_{n+1}=P y_{n}+Q y_{n-k}+R y_{n-l}+\frac{b y_{n-k}}{d y_{n-k}-e y_{n-l}} .
$$

Elsayed and El-Dessoky [45] investigated behavior of the rational difference equation of the fourth order

$$
x_{n+1}=a x_{n}+\frac{b x_{n} x_{n-2}}{c x_{n-2}+d x_{n-3}} .
$$

In this paper we investigate the global asymptotic behavior and the form of the solutions of the solutions of the following recursive sequences

$$
x_{n+1}=\frac{x_{n-2} x_{n-3}}{x_{n}\left( \pm 1 \pm x_{n-2} x_{n-3}\right)},
$$

where the initial conditions $x_{-3}, x_{-2}, x_{-1}$ and $x_{0}$ are arbitrary real numbers.

Here, we will review some of the definitions and theorems used in solving special cases of difference equations:

Definition 1.1. Let I be some interval of real numbers and let

$$
F: I^{k+1} \rightarrow I
$$

be a continuously differentiable function. Then for every set of initial condition $x_{-k}, x_{-k+1}, \ldots, x_{0} \in I$, the difference equation

$$
x_{n+1}=F\left(x_{n}, x_{n-1}, x_{n-2}, \ldots, x_{n-k}\right), \quad n=0,1, \ldots,
$$

has a unique solution $\left\{x_{n}\right\}_{n=-k}^{\infty}$. 
Definition 1.2. A point $x^{*} \in I$ is called an equilibrium point of (1.1) if

$$
x^{*}=F\left(x^{*}\right),
$$

that is,

$$
x_{n}=x^{*} \text { for all } n \geq-k .
$$

is a solution of (1.1), or equivalently, $x^{*}$ is a fixed point of $F$.

\section{Definition 1.3. (Stability)}

Let $x^{*}$ be an equilibrium point of (1.1).

(i) The equilibrium point $x^{*}$ of (1.1) is called locally stable if for every $\varepsilon>0$, there exists $\delta>0$ such that for all $\left\{x_{n}\right\}_{n=-k}^{\infty}$ is a solution of (1.1) and

$$
\left|x_{-k}-x^{*}\right|+\left|x_{1-k}-x^{*}\right|+\ldots+\left|x_{0}-x^{*}\right|<\delta,
$$

then

$$
\left|x_{n}-x^{*}\right|<\varepsilon \quad \text { for all } \quad n \geq 0 .
$$

(ii) The equilibrium point $x^{*}$ of (1.1) is called locally asymptotically stable if it is locally stable, and if there exists $\gamma>0$ such that if $\left\{x_{n}\right\}_{n=-k}^{\infty}$ is a solution of (1.1) and

$$
\left|x_{-k}-x^{*}\right|+\left|x_{-k+1}-x^{*}\right|+\ldots+\left|x_{0}-x^{*}\right|<\gamma
$$

then

$$
\lim _{n \rightarrow \infty} x_{n}=x^{*}
$$

(iii) The equilibrium point $x^{*}$ of (1.1) is called a global attractor iffor every solution $\left\{x_{n}\right\}_{n=-k}^{\infty}$ of (1.1) we have

$$
\lim _{n \rightarrow \infty} x_{n}=x^{*}
$$

(iv) The equilibrium point $x^{*}$ of (1.1) is called globally asymptotically stable if it is locally stable and global attractor of

(v) The equilibrium point $x^{*}$ of (1.1) is called unstable if $x^{*}$ is not locally stable.

\section{Linearized stability analysis}

Suppose that the function $F$ is continuously differentiable in some open neighborhood of an equilibrium point $x^{*}$. Let

$$
p_{i}=\frac{\partial F}{\partial u_{i}}\left(x^{*}, x^{*}, \ldots, x^{*}\right) \quad \text { for } i=0,1, \ldots, k,
$$

denote the partial derivatives of $F\left(u_{0}, u_{1}, \ldots . u_{k}\right)$ evaluated at the equilibrium $x^{*}$ of (1.1).

Then the equation

$$
y_{n+1}=p_{0} y_{n}+p_{1} y_{n-1}+\ldots+p_{k} y_{n-k}, \quad n=0,1, \ldots,
$$

is called the linearized equation associated of (1.1) about the equilibrium point $x^{*}$ and the equation

$$
\lambda^{k+1}-p_{0} \lambda^{k}-\ldots-p_{k-1} \lambda-p_{k}=0
$$

is called the characteristic equation of (2.1) about $x^{*}$.

The following result known as the Linear Stability Theorem is very useful in determining the local stability character of the equilibrium point $x^{*}$ of (1.1).

Theorem 2.1. [46] Assume that $p_{0}, p_{2}, \ldots, p_{k}$ are real numbers such that

$$
\left|p_{0}\right|+\left|p_{1}\right|+\ldots+\left|p_{k}\right|<1
$$

or

$$
\sum_{i=1}^{k}\left|p_{i}\right|<1
$$

Then all roots of (2.2) lie inside the unit disk. 


\section{Qualitative behavior of solutions of $x_{n+1}=\frac{x_{n-2} x_{n-3}}{x_{n}\left(1+x_{n-2} x_{n-3}\right)}$}

In this part, we study the some qualitative properties for the recursive equation in the form:

$$
x_{n+1}=\frac{x_{n-2} x_{n-3}}{x_{n}\left(1+x_{n-2} x_{n-3}\right)},
$$

where the initial values $x_{-3}, x_{-2}, x_{-1}$ and $x_{0}$ are arbitrary positive real numbers.

Theorem 3.1. Let $\left\{x_{n}\right\}_{n=-3}^{\infty}$ be a solution of difference equation (3.1). Then for $n=0,1, \ldots$

$$
\begin{aligned}
x_{6 n-3} & =d \prod_{i=0}^{n-1} \frac{(1+2 i c d)}{(1+(2 i+1) c d)} \frac{(1+2 i a b)}{(1+(2 i+1) a b)} \frac{(1+(2 i+1) b c)}{(1+2 i b c)}, \\
x_{6 n-2} & =c \prod_{i=0}^{n-1} \frac{(1+(2 i+1) c d)}{(1+(2 i+2) c d)} \frac{(1+(2 i+1) a b)}{(1+2 i a b)} \frac{(1+2 i b c)}{(1+(2 i+1) b c)}, \\
x_{6 n-1} & =b \prod_{i=0}^{n-1} \frac{(1+(2 i+2) c d)}{(1+(2 i+1) c d)} \frac{(1+2 i a b)}{(1+(2 i+1) a b)} \frac{(1+(2 i+1) b c)}{(1+(2 i+2) b c)}, \\
x_{6 n} & =a \prod_{i=0}^{n-1} \frac{(1+(2 i+1) c d)}{(1+(2 i+2) c d)} \frac{(1+(2 i+1) a b)}{(1+(2 i+2) a b)} \frac{(1+(2 i+2) b c)}{(1+(2 i+1) b c)}, \\
x_{6 n+1} & =\frac{c d}{a(1+c d)} \prod_{i=0}^{n-1} \frac{(1+(2 i+2) c d)}{(1+(2 i+3) c d)} \frac{(1+(2 i+2) a b)}{(1+(2 i+1) a b)} \frac{(1+(2 i+1) b c)}{(1+(2 i+2) b c)}, \\
x_{6 n+2}= & \frac{a b(1+c d)}{d(1+b c)} \prod_{i=0}^{n-1} \frac{(1+(2 i+3) c d)}{(1+(2 i+2) c d)} \frac{(1+(2 i+1) a b)}{(1+(2 i+2) a b)} \frac{(1+(2 i+2) b c)}{(1+(2 i+3) b c)},
\end{aligned}
$$

where $x_{-3}=d, x_{-2}=c, x_{-1}=b, x_{0}=a$.

Proof. For $n=0$, the result holds. Now, assume that $n>0$ and that our assumption holds for $n-1$. That is,

$$
\begin{aligned}
& x_{6 n-9}=d \prod_{i=0}^{n-2} \frac{(1+2 i c d)}{(1+(2 i+1) c d)} \frac{(1+2 i a b)}{(1+(2 i+1) a b)} \frac{(1+(2 i+1) b c)}{(1+2 i b c)}, \\
& x_{6 n-8}=c \prod_{i=0}^{n-2} \frac{(1+(2 i+1) c d)}{(1+(2 i+2) c d)} \frac{(1+(2 i+1) a b)}{(1+2 i a b)} \frac{(1+2 i b c)}{(1+(2 i+1) b c)}, \\
& x_{6 n-7}=b \prod_{i=0}^{n-2} \frac{(1+(2 i+2) c d)}{(1+(2 i+1) c d)} \frac{(1+2 i a b)}{(1+(2 i+1) a b)} \frac{(1+(2 i+1) b c)}{(1+(2 i+2) b c)}, \\
& x_{6 n-6}=a \prod_{i=0}^{n-2} \frac{(1+(2 i+1) c d)}{(1+(2 i+2) c d)} \frac{(1+(2 i+1) a b)}{(1+(2 i+2) a b)} \frac{(1+(2 i+2) b c)}{(1+(2 i+1) b c)}, \\
& x_{6 n-5}=\frac{c d}{a(1+c d)} \prod_{i=0}^{n-2} \frac{(1+(2 i+2) c d)}{(1+(2 i+3) c d)} \frac{(1+(2 i+2) a b)}{(1+(2 i+1) a b)} \frac{(1+(2 i+1) b c)}{(1+(2 i+2) b c)}, \\
& x_{6 n-4}=\frac{a b(1+c d)}{d(1+b c)} \prod_{i=0}^{n-2} \frac{(1+(2 i+3) c d)}{(1+(2 i+2) c d)} \frac{(1+(2 i+1) a b)}{(1+(2 i+2) a b)} \frac{(1+(2 i+2) b c)}{(1+(2 i+3) b c)} .
\end{aligned}
$$

From (3.1) that 


$$
\begin{aligned}
& x_{6 n-3}=\frac{x_{6 n-6} x_{6 n-7}}{x_{6 n-4}\left(1+x_{6 n-6} x_{6 n-7}\right)} \\
& a b \prod_{i=0}^{n-2}\left(\frac{1+2 i a b}{1+(2 i+2) a b}\right)
\end{aligned}
$$

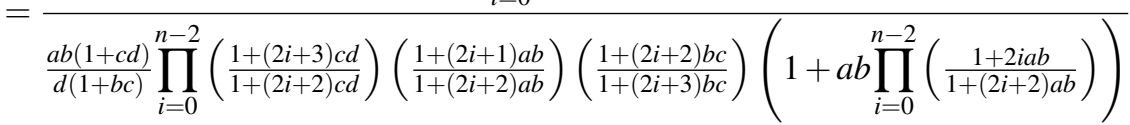

$$
\begin{aligned}
& =\frac{d(1+b c)}{(1+c d)} \frac{\left[\frac{(1+2 a b)(1+4 a b) \ldots(1+(2 n-6) a b)(1+2(n-2) a b)}{(1+2 a b)(1+4 a b) \ldots(1+(2 n-4) a b(1+(2 n-2) a b)}\right]}{\prod_{i=0}^{n-2}\left(\frac{1+(2 i+3) c d}{1+(2 i+2) c d}\right)\left(\frac{1+(2 i+1) a b}{1+(2 i+2) a b}\right)\left(\frac{1+(2 i+2) b c}{1+(2 i+3) b c}\right)\left(1+\frac{a b}{1+(2 n-2) a b}\right)} \\
& =d \frac{\left(\frac{1}{1+(2 n-2) a b}\right)}{\prod_{i=0}^{n-1}\left(\frac{1+(2 i+1) c d}{1+2 i c d}\right)\left(\frac{1+2 i b c}{1+(2 i+1) b c}\right) \prod_{i=0}^{n-2}\left(\frac{1+(2 i+1) a b}{1+(2 i+2) a b}\right)\left(\frac{1+(2 n-1) a b}{1+(2 n-2) a b}\right)} \\
& =\frac{d}{(1+(2 n-1) a b) \prod_{i=0}^{n-1}\left(\frac{1+(2 i+1) c d}{1+2 i c d}\right)\left(\frac{1+2 i b c}{1+(2 i+1) b c}\right) \prod_{i=0}^{n-2}\left(\frac{1+(2 i+1) a b}{1+(2 i+2) a b}\right)} \\
& =\frac{d}{\prod_{i=0}^{n-1}\left(\frac{1+(2 i+1) c d}{1+2 i c d}\right)\left(\frac{1+2 i b c}{1+(2 i+1) b c}\right)\left(\frac{1+(2 i+1) a b}{1+2 i a b}\right)} .
\end{aligned}
$$

Consequently, we have

$$
x_{6 n-3}=d \prod_{i=0}^{n-1}\left(\frac{1+2 i c d}{1+(2 i+1) c d}\right)\left(\frac{1+2 i a b}{1+(2 i+1) a b}\right)\left(\frac{1+(2 i+1) b c}{1+2 i b c}\right) .
$$

Similarly, other relations can be obtained and thus, the proof has been proved.

Theorem 3.2. The difference equation (3.1) has a unique equilibrium point which is 0 and it is not locally asymptotically stable.

Proof. For the equilibrium points of (3.1), is given by

$$
\begin{aligned}
x^{*} & =\frac{x^{*^{2}}}{x^{*}\left(1+x^{*^{2}}\right)}, \\
1+x^{*^{2}} & =1,
\end{aligned}
$$

or

$$
x^{*^{2}}=0 \text {. }
$$

Then the unique equilibrium point is $x^{*}=0$.

Let $F$ be function defined by

$$
F(u, v, w)=\frac{v w}{u(1+v w)} .
$$

Therefore it follows that

$$
F_{u}(u, v, w)=\frac{-v w}{u^{2}(1+v w)}, F_{v}(u, v, w)=\frac{w}{u(1+v w)^{2}}, F_{w}(u, v, w)=\frac{v}{u(1+v w)^{2}},
$$

we see that

$$
F_{u}\left(x^{*}, x^{*}, x^{*}\right)=-1, F_{v}\left(x^{*}, x^{*}, x^{*}\right)=1, F_{w}\left(x^{*}, x^{*}, x^{*}\right)=1 .
$$

This completes the proof by using Theorem 2.1 . 


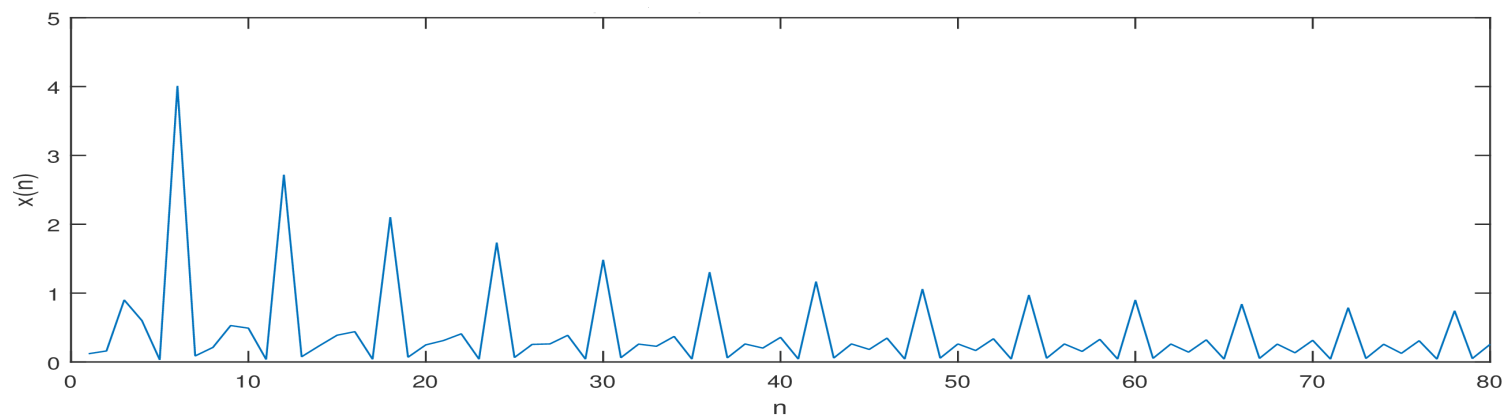

Figure 3.1. Plot of $x(n+1)=\frac{x(n-2) x(n-3)}{x(n)(1+x(n-2) x(n-3))}$

\section{Numerical Examples}

For statement the results of this part, we take into account numerical examples which illustrate different types of solutions to $(3.1)$.

Example 3.3. See Figure 3.1, since $x_{-3}=0.12, x_{-2}=0.16, x_{-1}=0.9$ and $x_{0}=0.6$

\section{Qualitative behavior of solutions of $x_{n+1}=\frac{x_{n-2} x_{n-3}}{x_{n}\left(-1+x_{n-2} x_{n-3}\right)}$}

Here, we obtain the solution of the following difference equation

$$
x_{n+1}=\frac{x_{n-2} x_{n-3}}{x_{n}\left(-1+x_{n-2} x_{n-3}\right)}, \quad n=0,1, \ldots
$$

where the initial conditions $x_{-3}, x_{-2}, x_{-1}$ and $x_{0}$.are arbitrary real numbers with $x_{-2} x_{-3} \neq 1, x_{-1} x_{-2} \neq 1$ and $x_{0} x_{-1} \neq 1$.

Theorem 4.1. Let $\left\{x_{n}\right\}_{n=-3}^{\infty}$ be a solution of difference equation of (4.1). Then the equation (4.1) has unboundedness solutions and for $n=0,1, \ldots$

$$
\begin{aligned}
& x_{6 n-3}=\frac{d(-1+b c)^{n}}{(-1+c d)^{n}(-1+a b)^{n}}, \quad x_{6 n-2}=\frac{c(-1+c d)^{n}(-1+a b)^{n}}{(-1+b c)^{n}}, \\
& x_{6 n-1}=\frac{b(-1+b c)^{n}}{(-1+c d)^{n}(-1+a b)^{n}}, \quad x_{6 n}=\frac{a(-1+c d)^{n}(-1+a b)^{n}}{(-1+b c)^{n}}, \\
& x_{6 n+1}=\frac{c d(-1+b c)^{n}}{a(-1+a b)^{n}(-1+c d)^{n+1}}, \quad x_{6 n+2}=\frac{a b(-1+c d)^{n+1}(-1+a b)^{n}}{d(-1+b c)^{n+1}},
\end{aligned}
$$

where $x_{-3}=d, x_{-2}=c, x_{-1}=b$ and $x_{0}=a$.

Proof. For $n=0$ the conclusion holds. Now, assume that $n>0$ and that our assumption holds for $n-1$. That is,

$$
\begin{aligned}
& x_{6 n-9}=\frac{d(-1+b c)^{n-1}}{(-1+c d)^{n-1}(-1+a b)^{n-1}}, \quad x_{6 n-8}=\frac{c(-1+c d)^{n-1}(-1+a b)^{n-1}}{(-1+b c)^{n-1}}, \\
& x_{6 n-7}=\frac{b(-1+b c)^{n-1}}{(-1+c d)^{n-1}(-1+a b)^{n-1}}, \quad x_{6 n-6}=\frac{a(-1+c d)^{n-1}(-1+a b)^{n-1}}{(-1+b c)^{n-1}}, \\
& x_{6 n-5}=\frac{c d(-1+b c)^{n-1}}{a(-1+a b)^{n-1}(-1+c d)^{n}}, \quad x_{6 n-4}=\frac{a b(-1+c d)^{n}(-1+a b)^{n-1}}{d(-1+b c)^{n}} .
\end{aligned}
$$

Now we proof some of relations of (4.2). 


$$
\begin{aligned}
x_{6 n-2} & =\frac{x_{6 n-5} x_{6 n-6}}{x_{6 n-3}\left(-1+x_{6 n-5} x_{6 n-6}\right)}=\frac{(c d)(-1+c d)^{-1}}{\frac{d(-1+b c)^{n}}{(-1+c d)^{n}(-1+a b)^{n}}\left(-1+c d(-1+c d)^{-1}\right)} \\
& =\frac{c(-1+c d)^{n-1}(-1+a b)^{n}}{(-1+b c)^{n}\left(-1+\frac{c d}{(-1+c d)}\right)}=\frac{c(-1+c d)^{n}(-1+a b)^{n}}{(-1+b c)^{n}} .
\end{aligned}
$$

Also,

$$
\begin{aligned}
x_{6 n-1} & =\frac{x_{6 n-4} x_{6 n-5}}{x_{6 n-2}\left(-1+x_{6 n-4} x_{6 n-5}\right)} \\
& =\frac{\left(b c(-1+b c)^{n-1}\right) l(-1+b c)^{n}}{\left(c(-1+c d)^{n}(-1+a b)^{n} /(-1+b c)^{n}\right)\left(-1+\frac{b c}{-1+b c}\right)} \\
& =\frac{b(-1+b c)^{n-1}}{(-1+c d)^{n}(-1+a b)^{n}\left(\frac{1}{-1+b c}\right)}=\frac{b(-1+b c)^{n}}{(-1+c d)^{n}(-1+a b)^{n}}
\end{aligned}
$$

Similarly, other relations can be obtained and thus, the proof has been proved.

Theorem 4.2. The difference equation (4.1) has a periodic solution of periodic six iff $a b=2$ and $b=d$ and we will take the form:

$$
\left\{d, c, b, a, \frac{c d}{a(-1+c d)}, \frac{a b}{d}, d, c, b, a, \frac{c d}{a(-1+c d)}, \frac{a b}{d}, \ldots\right\} .
$$

Proof. Assume that there exists a prime period six solution of (4.1):

$$
d, c, b, a, \frac{c d}{a(-1+c d)}, \frac{a b}{d}, d, c, b, a, \frac{c d}{a(-1+c d)}, \frac{a b}{d}, \ldots
$$

From (4.2), we get

$$
\begin{aligned}
& x_{6 n-3}=d=\frac{d(-1+b c)^{n}}{(-1+c d)^{n}(-1+a b)^{n}}, \quad x_{6 n-2}=c=\frac{c(-1+c d)^{n}(-1+a b)^{n}}{(-1+b c)^{n}}, \\
& x_{6 n-1}=b=\frac{b(-1+b c)^{n}}{(-1+c d)^{n}(-1+a b)^{n}}, \quad x_{6 n}=a=\frac{a(-1+c d)^{n}(-1+a b)^{n}}{(-1+b c)^{n}} \\
& x_{6 n+1}=\frac{c d(-1+b c)^{n}}{a(-1+c d)}=\frac{c d(-1+a b)^{n}(-1+c d)^{n+1}}{a(-1+} \\
& x_{6 n+2}=\frac{a b}{d}=\frac{a b(-1+c d)^{n+1}(-1+a b)^{n}}{d(-1+b c)^{n+1}} .
\end{aligned}
$$

Then we can see that

$$
a b=2 \text { and } b=d .
$$


Conversely, suppose that $a b=2$ and $b=d$. Then we see that

$$
\begin{aligned}
x_{6 n-3} & =\frac{d(-1+b c)^{n}}{(-1+c d)^{n}(-1+a b)^{n}}=\frac{d(-1+c d)^{n}}{(-1+c d)^{n}(-1+2)^{n}}=d, \\
x_{6 n-2} & =\frac{c(-1+c d)^{n}(-1+a b)^{n}}{(-1+b c)^{n}}=\frac{c(-1+c d)^{n}(-1+2)^{n}}{(-1+c d)^{n}}=c, \\
x_{6 n-1} & =\frac{b(-1+b c)^{n}}{(-1+c d)^{n}(-1+a b)^{n}}=\frac{b(-1+c d)^{n}}{(-1+c d)^{n}(-1+2)^{n}}=b, \\
x_{6 n} & =\frac{a(-1+c d)^{n}(-1+a b)^{n}}{(-1+b c)^{n}}=a, \\
x_{6 n+1} & =\frac{c d(-1+b c)^{n}}{a(-1+a b)^{n}(-1+c d)^{n+1}}=\frac{c d}{a(-1+c d)}, \\
x_{6 n+2} & =\frac{a b(-1+c d)^{n+1}(-1+a b)^{n}}{d(-1+b c)^{n+1}}=\frac{a b}{d} .
\end{aligned}
$$

Thus we obtained a periodic solution of period six.

Theorem 4.3. Equation (4.1) has a periodic solution of period two iff $a b=b c=c d=2$ ( It also means $a=c, b=d$ ) and we will take the form:

$$
\{d, c, d, c, \ldots\} .
$$

Proof. First assume that there exists a prime period two solution of (4.1):

$$
d, c, d, c, \ldots .
$$

We see from the form of the solutions of (4.1) that

$$
\begin{aligned}
& x_{6 n-3}=d=\frac{d(-1+b c)^{n}}{(-1+c d)^{n}(-1+a b)^{n}}, \quad x_{6 n-2}=c=\frac{c(-1+c d)^{n}(-1+a b)^{n}}{(-1+b c)^{n}}, \\
& x_{6 n-1}=d=\frac{b(-1+b c)^{n}}{(-1+c d)^{n}(-1+a b)^{n}}, \quad x_{6 n}=c=\frac{a(-1+c d)^{n}(-1+a b)^{n}}{(-1+b c)^{n}}, \\
& x_{6 n+1}=d=\frac{c d(-1+b c)^{n}}{a(-1+a b)^{n}(-1+c d)^{n+1}}, \quad x_{6 n+2}=c=\frac{a b(-1+c d)^{n+1}(-1+a b)^{n}}{d(-1+b c)^{n+1}} .
\end{aligned}
$$

Thus we see that $a b=b c=c d=2$.

Second suppose that $a b=b c=c d=2$. Then we obtain

$$
\begin{aligned}
x_{6 n-3} & =\frac{d(-1+b c)^{n}}{(-1+c d)^{n}(-1+a b)^{n}}=\frac{d(-1+2)^{n}}{(-1+2)^{n}(-1+2)^{n}}=d, \\
x_{6 n-2} & =\frac{c(-1+c d)^{n}(-1+a b)^{n}}{(-1+b c)^{n}}=\frac{c(-1+2)^{n}(-1+2)^{n}}{(-1+2)^{n}}=c, \\
x_{6 n-1} & =\frac{b(-1+b c)^{n}}{(-1+c d)^{n}(-1+a b)^{n}}=\frac{b(-1+2)^{n}}{(-1+2)^{n}(-1+2)^{n}}=d, \\
x_{6 n} & =\frac{a(-1+c d)^{n}(-1+a b)^{n}}{(-1+b c)^{n}}=c, \\
x_{6 n+1} & =\frac{c d(-1+b c)^{n}}{a(-1+a b)^{n}(-1+c d)^{n+1}}=d, \\
x_{6 n+2} & =\frac{a b(-1+c d)^{n+1}(-1+a b)^{n}}{d(-1+b c)^{n+1}}=c .
\end{aligned}
$$

Thus we obtained a periodic solution of period two. 


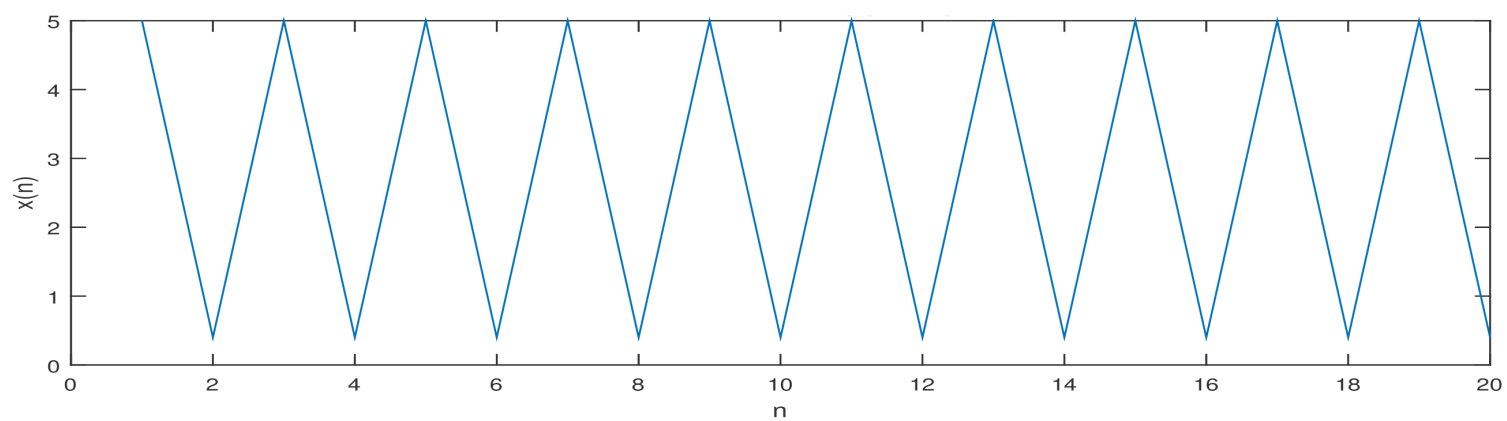

Figure 4.1. Plot of $x(n+1)=\frac{x(n-2) x(n-3)}{x(n)(-1+x(n-2) x(n-3))}$

Theorem 4.4. Difference equation (4.1) has equilibrium points which are $0, \pm \sqrt{2}$ such that they are not locally asymptotically stable.

Proof. We can write

$$
x^{*}=\frac{x^{*^{2}}}{x^{*}\left(-1+x^{*^{2}}\right)}, \text { or } x^{* 2}\left(x^{* 2}-2\right)=0,
$$

consequently $0, \pm \sqrt{2}$ are the equilibrium points.

Suppose that $F:(0, \infty)^{3} \rightarrow(0, \infty)$ be function defined by

$$
F(u, v, w)=\frac{v w}{u(-1+v w)},
$$

then

$$
F_{u}(u, v, w)=\frac{-v w}{u^{2}(-1+v w)}, F_{v}(u, v, w)=\frac{-w}{u(-1+v w)^{2}}, F_{w}(u, v, w)=\frac{-v}{u(-1+v w)^{2}},
$$

we see that,

$$
F_{u}\left(x^{*}, x^{*}, x^{*}\right)=-1, F_{v}\left(x^{*}, x^{*}, x^{*}\right)=-1, F_{w}\left(x^{*}, x^{*}, x^{*}\right)=-1 .
$$

This completes the proof by using Theorem 2.1.

\section{Numerical Examples}

We put some numerical examples which illustrate different types of solutions of (4.1).

Example 4.5. When we put $x_{-3}=5, x_{-2}=2 / 5, x_{-1}=5$ and $x_{0}=2 / 5$. See Figure 4.1.

\section{Qualitative behavior of solutions of $x_{n+1}=\frac{x_{n-2} x_{n-3}}{x_{n}\left(1-x_{n-2} x_{n-3}\right)}$}

In this section, we get the expressions of the solution of the difference equation in the form:

$$
x_{n+1}=\frac{x_{n-2} x_{n-3}}{x_{n}\left(1-x_{n-2} x_{n-3}\right)}, \quad n=0,1, \ldots
$$

where the initial conditions $x_{-3}, x_{-2}, x_{-1}$ and $x_{0}$ are arbitrary real numbers.

Theorem 5.1. Let $\left\{x_{n}\right\}_{n=-3}^{\infty}$ be a solution of the difference equation of (5.1). Then for $n=0,1, \ldots$ 


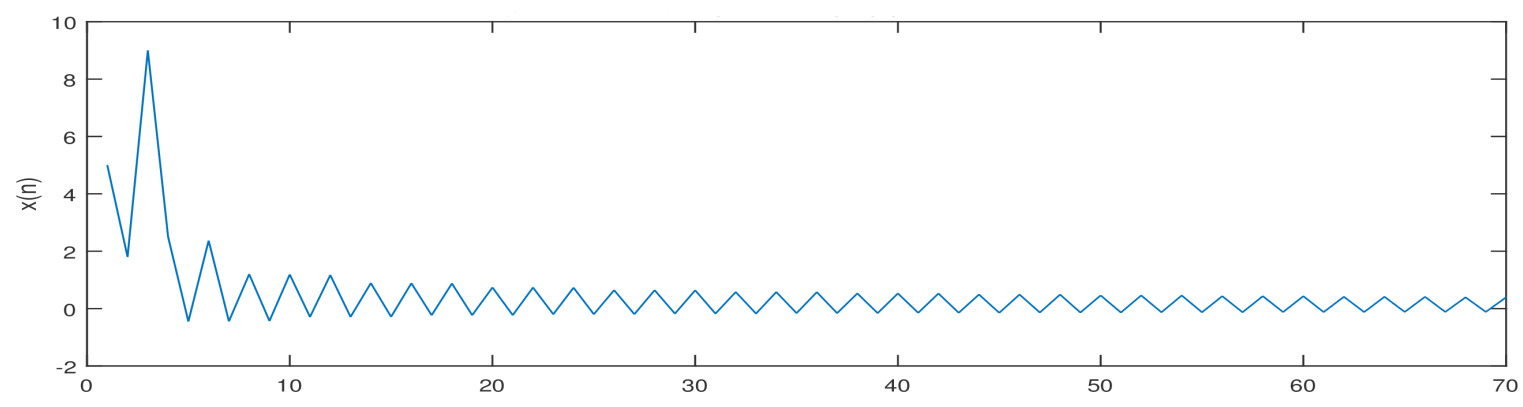

Figure 5.1. Plot of $x(n+1)=\frac{{ }^{n}}{x(n)(1-2) x(n-2) x(n-3))}$

$$
\begin{aligned}
x_{6 n-3} & =d \prod_{i=0}^{n-1} \frac{(1-2 i c d)}{(1-(2 i+1) c d)} \frac{(1-2 i a b)}{(1-(2 i+1) a b)} \frac{(1-(2 i+1) b c)}{(1-2 i b c)}, \\
x_{6 n-2} & =c \prod_{i=0}^{n-1} \frac{(1-(2 i+1) c d)}{(1-(2 i+2) c d)} \frac{(1-(2 i+1) a b)}{(1-2 i a b)} \frac{(1-2 i b c)}{(1-(2 i+1) b c)}, \\
x_{6 n-1} & =b \prod_{i=0}^{n-1} \frac{(1-(2 i+2) c d)}{(1-(2 i+1) c d)} \frac{(1-2 i a b)}{(1-(2 i+1) a b)} \frac{(1-(2 i+1) b c)}{(1-(2 i+2) b c)}, \\
x_{6 n} & =a \prod_{i=0}^{n-1} \frac{(1-(2 i+1) c d)}{(1-(2 i+2) c d)} \frac{(1-(2 i+1) a b)}{(1-(2 i+2) a b)} \frac{(1-(2 i+2) b c)}{(1-(2 i+1) b c)} \\
x_{6 n+1} & =\frac{c d}{a(1-c d)} \prod_{i=0}^{n-1} \frac{(1-(2 i+2) c d)}{(1+(2 i+3) c d)} \frac{(1-(2 i+2) a b)}{(1+(2 i+1) a b)} \frac{(1-(2 i+1) b c)}{(1+(2 i+2) b c)}, \\
x_{6 n+2} & =\frac{a b(1-c d)}{d(1-b c)} \prod_{i=0}^{n-1} \frac{(1-(2 i+3) c d)}{(1-(2 i+2) c d)} \frac{(1-(2 i+1) a b)}{(1-(2 i+2) a b)} \frac{(1-(2 i+2) b c)}{(1-(2 i+3) b c)} .
\end{aligned}
$$

Theorem 5.2. Equation (5.1) has a unique equilibrium point which is 0 and it is not locally asymptotically stable.

Example 5.3. See Figure 5.1, we let $x_{-3}=5, x_{-2}=1.8, x_{-1}=9$ and $x_{-0}=2.5$.

\section{Qualitative behavior of solutions of $x_{n+1}=\frac{x_{n-2} x_{n-3}}{x_{n}\left(-1-x_{n-2} x_{n-3}\right)}$}

In this part, we obtain the form of solution of the following difference equation

$$
x_{n+1}=\frac{x_{n-2} x_{n-3}}{x_{n}\left(-1-x_{n-2} x_{n-3}\right)}, \quad n=0,1, \ldots
$$

where the initial conditions $x_{-3}, x_{-2}, x_{-1}$ and $x_{0}$ are arbitrary real numbers with $x_{-2} x_{-3} \neq-1, x_{-1} x_{-2} \neq-1$ and $x_{0} x_{-1} \neq-1$.

Theorem 6.1. Let $\left\{x_{n}\right\}_{n=-3}^{\infty}$ be a solution of (6.1). Then (6.1) has the following solution for $n=0,1, \ldots$

$$
\begin{aligned}
& x_{6 n-3}=\frac{d(-1-b c)^{n}}{(-1-c d)^{n}(-1-a b)^{n}}, \quad x_{6 n-2}=\frac{c(-1-c d)^{n}(-1-a b)^{n}}{(-1-b c)^{n}}, \\
& x_{6 n-1}=\frac{b(-1-b c)^{n}}{(-1-c d)^{n}(-1-a b)^{n}}, \quad x_{6 n}=\frac{a(-1-c d)^{n}(-1-a b)^{n}}{(-1-b c)^{n}}, \\
& x_{6 n+1}=\frac{c d(-1-b c)^{n}}{a(-1-a b)^{n}(-1-c d)^{n+1}}, \quad x_{6 n+2}=\frac{a b(-1-c d)^{n+1}(-1-a b)^{n}}{d(-1-b c)^{n+1}} .
\end{aligned}
$$

Theorem 6.2. Difference equation (6.1) has a periodic solution of period six iff $a b=-2$ and $b=d$ and we will take the form:

$$
\left\{d, c, b, a, \frac{c d}{a(-1-c d)}, \frac{a b}{d}, d, c, b, a, \frac{c d}{a(-1-c d)}, \frac{a b}{d}, \ldots\right\} .
$$




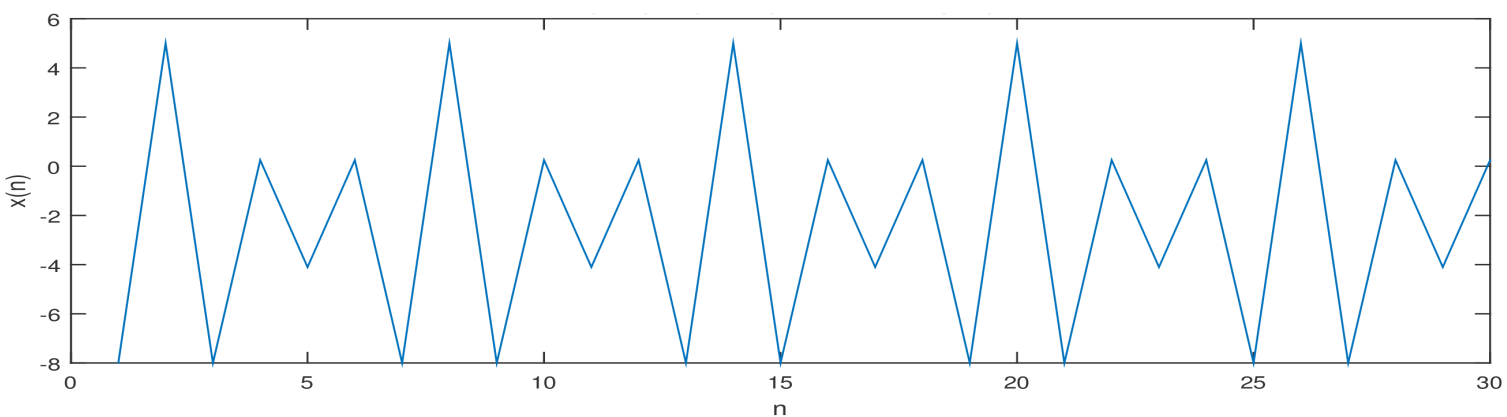

Figure 6.1. Plot of $x(n+1)=\frac{x(n-2) x(n-3)}{x(n)(-1-x(n-2) x(n-3))}$

Theorem 6.3. Equation (6.1) has a periodic solution of period two iff $a b=b c=c d=-2$ and takes the form: $\{d, c, d, c, \ldots\}$.

Theorem 6.4. Difference equation (6.1) has equilibrium point which is 0 and it is not locally asymptotically stable.

Example 6.5. Figure 6.1 shows the period six solutions of (6.1) since $x_{-3}=-8, x_{-2}=5, x_{-1}=-8$ and $x_{0}=1 / 4$.

\section{Acknowledgements}

This article was funded by the Deanship of Scientific Research (DSR), King Abdulaziz University. The authors, therefore, acknowledge with thanks to DSR technical and financial support.

\section{References}

[1] V. L. Kocic, G. Ladas, Global Behavior of Nonlinear Difference Equations of Higher Order with Applications, Kluwer Academic Publishers, Dordrecht, 1993.

[2] A. Asiri, M. M. El-Dessoky, E. M. Elsayed, Solution of a third order fractional system of difference equations, J. Comput. Anal. Appl., 24 (3) (2018), 444-453.

[3] E. Camouzis, G. Ladas, Dynamics of Third-Order Rational Difference Equations with Open Problems and Conjectures, Chapman \& Hall/CRC, Boca Raton, Fla, USA, 2004.

${ }^{[4]}$ Q. Din, Global Stability and Neimark-Sacker bifurcation of a host-parasitoid model, Int. J. Syst. Sct., 48 (6) (2017), 1194-1202.

[5] Q. Din, E. M. Elsayed, Stability analysis of a discrete ecological model, Computational Ecology and Software, 2 (2014), 89-103.

[6] Q. Din, M. A. Khan, Global stability of a system of exponential difference equations, CMMPG, 1 (2) (2016), 71-85.

${ }^{\text {[7] }}$ Q. Din, M. A. Khan, U. Saeed, Qualitative behaviour of generalised Beddington model, Zeitschrift für Naturforschung A, 71 (2) (2016), 145-155.

${ }^{[8]}$ E. M. Elabbasy, A. A. Elsadany, S. Ibrahim, Qualitative behavior of rational difference equations of Higher Order, Malaya J. Mat., 3 (4) (2015), 530-539.

[9] H. El-Metwally, E. M. Elsayed, Qualitative Behavior of some rational difference equations, J. Comput. Anal. Appl., 20 (2) (2016), 226-236.

[10] M. A. El-Moneam, On The dynamics of the solutions of the rational recursive sequences, Brit. J. Math. Comput. Sci., 5 (2015), 654-665.

[11] M. A. El-Moneam, S. O. Alamoudy, On study of the asymptotic behavior of some rational difference equations, DCDIS, A: Mathematical Analysis, 22 (2015), 157-176.

${ }^{[12]}$ E. M. Elsayed, On the solutions and periodicity of some rational systems of difference equations, B. Math. Soc. Sci. Math., 60 (108) (2) (2017), 159-171.

${ }^{[13]}$ E. M. Elsayed, A. Khaliq, Global attractivity and periodicity behavior of a recursive sequence, J. Comput. Anal. Appl., 22 (2) (2017), 369-379. 
[14] E. M. Elsayed, A. M. Ahmed, Dynamics of a three-dimensional systems of rational difference equations, Math. Method Appl. Sci., 39 (5) (2016), 1026-1038.

[15] E. M. Elsayed, A. Alghamdi, Dynamics and global stability of higher order nonlinear difference equation, J. Comput. Anal. Appl., 21 (3) (2016), 493-503.

[16] E. M. Elsayed, M. M. El-Dessoky, Dynamics and global behavior for a fourth-order rational difference equation, Hacet. J. Math. Stat., 42 (5) (2013), 479-494.

[17] E. M. Elsayed, M. Ghazel, A. E. Matouk, Dynamical analysis of the rational difference equation $x_{n+1}=C x_{n-3} /(A+$ $\left.B x_{n-1} x_{n-3}\right)$, J. Comput. Anal. Appl, 23 (3) (2017), 496-507.

[18] E. M. Elsayed, T. F. Ibrahim, Solutions and periodicity of a rational recursive sequences of order five, B. Malaysian Math. Sci. Soc., 38 (1) (2015), 95-112.

[19] E. M. Elsayed, T. F. Ibrahim, Periodicity and Solutions for Some Systems of Nonlinear Rational Difference Equations, Hacet. J. Math. Stat., 44 (6) (2015), 1361-1390.

[20] M. Erdogan, K. Uslu, Behavior of a Nonlinear Difference Equation $x_{n+1}=\frac{1-x_{n}}{A+\sum_{i=1}^{k} x_{n-i}}$, Journal of Life Sciences, 10 (2016), 215-219.

[21] T. F. Ibrahim, Periodicity and global attractivity of difference equation of higher order, J. Comput. Anal. Appl., 16 (2014), 552-564.

[22] T. F. Ibrahim, Solving a class of third-order max-type difference equations, DCDIS, Series A:Mathematical Analysis , 21(2014), 333-342.

[23] T. F. Ibrahim, Closed form expression of tractable semi-max-type two-dimensional system of difference equations with variable coefficients, JOEMS, 24 (4) (2016), 538-544.

[24] T. F. Ibrahim, M. A. El-Moneam, Global stability of a higher-order difference equation, Iran. J. Sci. Technol. A, 1 (41) (2017), 51-58

[25] R. Karatas, C. Cinar, D. Simsek, On positive solutions of the difference equation $x_{n+1}=\frac{x_{n-5}}{1+x_{n-2} x_{n-5}}$, Int. J. Contemp. Math. Sci., 1 (10) (2006) 495500.

[26] A. Q. Khan, Q. Din, M. N. Qureshi, T. F. Ibrahim, Global behavior of an anti-competitive system of fourth-order rational difference equations, Computational Ecology and Software, 2014, 4(1).

[27] K. Liu, P. Li, F. Han, W. Zhong, Global dynamics of nonlinear difference equation $x_{n+1}=A+x_{n} / x_{n-1} x_{n-2}$, J. Comput. Anal. Appl., 24 (6) (2018), 1125-1132.

[28] R. Mostafaei, N. Rastegar, On a recurrence relation, QScience Connect, 10 (2014), 1-11.

[29] U. Saeed, M. Ozair, T. Hussain, Q. Din, Fractional-order vector-host disease model, DCDIS, Series B: Applications \& Algorithms, 24 (2017), 97-111.

[30] H. Sedaghat, Nonlinear Difference Equations, Theory and Applications to Social Science Models, Kluwer Academic Publishers, Dordrecht, 2003.

[31] D. T. Tollou, Y. Yazlik, N. Taskara, Behavior of positive solutions of a difference equation, J. Appl. Math. \& Informatics, 35 (3-4) (2017), 217 - 230.

[32] N. Touafek, On a second order rational difference equation, Hacet. J. Math. Stat., 41 (6) (2012), 867-874.

[33] C. Wang, M. Hu, On the solutions of a rational recursive sequence, JMI, 1 (2013), 25-33.

[34] C. Y. Wang, X. J. Fang, R. Li, On the dynamics of a certain four-order fractional difference equations, J. Comput. Anal. Appl., 22 (5) (2017), 968-976.

[35] Y. Yazlik, E. M. Elsayed, N. Taskara, On the behaviour of the solutions of difference equation systems, J. Comput. Anal. Appl., 16 (5) (2014), 932-941.

[36] E. M. E. Zayed, Qualitative behavior of the rational recursive sequence $x_{n+1}=A x_{n}+B x_{n-k}+\frac{p+x_{n-k}}{q x_{n}+x_{n-k}}$, Inter. J. Adv. Math., 1 (1) (2014), 44-55.

[37] R. Karatas, Global behavior of a higher order difference equation, Inter. J. Contemp. Math. Sci., 12 (3) (2017), 133-138. 
[38] M. Gumus, R. Abo-Zeid, Ö. Öcalan, Dynamical behavior of a third-order difference equation with arbitrary powers, Kyungpook Math. J., 57 (2017), 251-263.

[39] E. M. Elsayed, Dynamics and behavior of a higher order rational difference equation, J. Nonlinear Sci. App., 9 (4) (2016), 1463-1474.

[40] M. R. S. Kulenovic, G. Ladas,N. R. Prokup, A rational difference Equation, Comput. Math. Appl., 41 (2001), 671-678.

[41] E. M. Elsayed, New method to obtain periodic solutions of period two and three of a rational difference equation, Nonlinear Dynam., 79 (1) (2015), 241-250.

[42] M. A. Al-Shabi, R. Abo-Zeid, Global asymptotic stability of a higher order difference equation, Appl. Math. Sci., 4(17) (2010), 839-847.

[43] A. M. Amleh, E. Drymonis, Eventual Monotonicity in Nonlinear Difference Equation, International Journal of Difference Equation, 11 (2) (2016), 123-137.

[44] S. Nirmaladevi, N. Karthikeyan, Dynamics and behavior of higher order nonlinear rational difference equation, IJARIIE, 3 (4) (2017), 2395-4396.

[45] E. M. Elsayed, H. El-Metwally, Global Behavior and periodicity of some difference equations, J. Comput. Anal. Appl, 19 (2) (2015), 298-309.

[46] M. Akbar, N. Ali, The improved F-expansion method with Riccati equation and its applications in mathematical physics, Cogent Math., 4 (1) (2017), 1-19. 